\title{
Joule per Mole-Kelvin
}

National Cancer Institute

\section{Source}

National Cancer Institute. Joule per Mole-Kelvin. NCI Thesaurus. Code C70449.

A SI unit of molar entropy used also as a unit of molar heat capacity. 